\title{
A Reference-Dependent Regret Model for Deterministic Trade-off Studies
}

\author{
Edouard Kujawski \\ Engineering Division \\ Lawrence Berkeley National Laboratory \\ Berkeley, California 94720 \\ E-mail: e_kujawski@lbl.gov \\ Telephone: (510) 486-6932
}

\begin{abstract}
Today's typical multi-criteria decision analysis is based on classical expected utility theory that assumes a mythical "Rational Individual" immune to psychological influences such as anticipated regret. It is therefore in conflict with rational individuals who trade-off some benefits and forgo the alternative with the highest total classical utility for a more balanced alternative in order to reduce their levels of anticipated regret. This paper focuses on decision making under certainty. It presents a reference-dependent regret model (RDRM) in which the level of regret that an individual experiences depends on the absolute values rather than the differences of the utilities of the chosen and forgone alternatives. The RDRM best choice may differ from the conventional linear additive utility model, the analytic hierarchy process, and the regret theory of Bell and Loomes and Sugden. Examples are presented that indicate that RDRM is the better predictive descriptor for decision making under certainty. RDRM satisfies transitivity of the alternatives under pairwise comparisons and models rank reversal consistent with observed reasonable choices under dynamic or distinct situations. Like regret theory, the RDRM utilities of all the alternatives under consideration are interrelated. For complex trade-off studies regret is incorporated as an element of a cost-utility-regret analysis that characterizes each alternative in terms of its monetary cost, an aggregate performance utility, and a regret value. This provides decision makers adequate information to compare the alternatives and depending on their values they may trade-off some performance and/or cost to avoid high levels of regret. The result is a well-balanced alternative often preferred by reasonable decision makers to the optimal choice of classical multi-attribute utility analysis. The model can readily be extended to incorporate rejoicing to suit decision makers who seek it. The approach is illustrated using a hypothetical but realistic aircraft selection problem.
\end{abstract}

Key words: multi-attribute utility analysis, multi-criteria decision making, regret theory, transitivity, rank reversal, preference reversal, cost-utility analysis, Pareto optimum, analytic hierarchy process 


\section{INTRODUCTION}

Most people have experienced or can imagine the feeling of regret associated with choosing an alternative from a set of alternatives when a forgone alternative possesses one or more preferred attributes or criteria. There is significant evidence from laboratory studies and real-world experience that humans often base their choices on comparisons across the alternatives under consideration and relative to "what might have been" under another choice [Plous, 1993; Hastie and Dawes, 2001]. Anticipated regret and its counterpart, rejoicing, tend to be important considerations in decision-making under both certainty and uncertainty. However, humans must check these emotions from becoming irrational forces that prevent them from coping well with complex decisions. With this perspective, a strategy such as minimax regret that is driven solely by regret may be considered deficient and/or irrational [Haszelrigg, 1996]. The classical Expected Utility Theory (EUT), which was introduced by Bernoulli almost 300 years ago and provided an axiomatic foundation by von Neumann and Morgenstern [1953], also has shortcomings because it assumes a mythical "Rational Individual" devoid of psychological influences or emotions [Luce, 1992]. In the past fifty years noted psychiatrists, economists, and decision theorists have concluded that classical EUT fails to account for important psychological, cultural and organizational aspects of decision-making under uncertainty. Prospect theory [Kahneman and Tversky, 1979; Tversky and Kahneman, 1992], rank-dependent utility [Quiggin, 1982; Diecidue and Wakker, 2001], and regret theory [Bell, 1982; Loomes and Sugden, 1982 and 1986] have become recognized as viable alternatives. We differentiate between the "Rational Individual" with a capital R and a capital C and the "rational individual" with lower case $r$ and lower case $i$. We use the expression "Rational Individual" to refer to the person who makes all decisions in accordance with classical EUT. We use the expression "rational individual" to refer to the person who uses reason and emotions to make decisions in a systematic and logical manner with due consideration to the personal and social context. The distinction between reason and emotion is problematic and the two often appear to be inseparable [Damasio, 1995]. For example, anticipated regret requires comparing the chosen and forgone alternatives.

In this paper we consider the Multi-Criteria Decision Analysis (MCDA) problem of choosing the "best alternative" from a finite set of deterministic alternatives characterized by multiple criteria that may be qualitative, quantified with different units of measure, and conflict with each other. Seldom does an alternative or solution exist that is as good as or better than the other alternatives for all criteria. A Decision-Maker (DM) then has to choose his "best choice" from a set of Pareto-optimal alternatives. Numerous methods have been developed for facilitating such decisions [Mollaghasemi and Pet-Edwards, 1997]. Each has its strengths and weaknesses. Even though they have been applied to thousands of real-world problems, there is little serious ex-post analysis of their usefulness and validity [Corner and Kirkwood 1991]. In this paper we focus on the method based on the notion of multi-attribute utility [Keeney and Raiffa, 1976]. We refer to it as Multi-Attribute Utility Analysis (MAUA). (Other commonly used names are multi-attribute value analysis [Buede, 2000] and multi-attribute utility theory [Clemen and Reilley, 2001].)

It is seldom explicitly acknowledged that today's typical MAUA includes the following assumptions and simplifications: 
1. Emotions are not considered beyond the subjective assessment of the utilities and weights. Specifically, an alternative's utilities do not depend on the other alternatives. This requires no comparisons across alternatives or an individual who does not experience emotions such as regret and rejoicing. The notion of regret-rejoicing is therefore in conflict with today's typical MAUA.

2. The utility of each criterion is independent of the other criteria. This property, known as mutual preferential independence, is problem and modeling specific.

3. Each alternative is characterized by a Multi-attribute Utility Function (MUF), $U\left(A_{i}\right)=U\left(u_{i 1}\right.$, $\left.u_{i 2}, . ., u_{i m}\right)$, such that $A_{i}$ is preferred to $A_{j}$ (we will denote it as $A_{i} \succ A_{j}$ ) if and only if $U\left(A_{i}\right)>$ $\mathrm{U}\left(\mathrm{A}_{\mathrm{j}}\right)$. The choice of a MUF plays a major role in the scoring and different ones can lead to conflicting results.

4. Serious doubts have been raised about the validity and usefulness of using a single number to characterize complex alternatives. Sharpe [1994: 57] states "Clearly, any measure that attempts to summarize even an unbiased prediction of performance with a single number requires a substantial set of assumptions for justifications."

Given the above assumptions and simplifications, it is no surprise that deviations from MAUA predictions cannot always be explained away as faulty data or a flawed analysis [See and Lewis, 2002]. MCDA is a very active field of research and applications. The basic concepts of utility theory, the adequacy of a MUF, and rationality are being revisited. Extensions, revisions, and new approaches are being developed [Lootsma, 2000].

This paper has two main thrusts that address the listed MAUA problems: the ReferenceDependent Regret Model (RDRM) and Cost-Utility-Regret Analysis (CURA). In RDRM, we assume that the level of regret that an individual experiences depends on the absolute values rather than simply the differences of the utilities of the chosen and forgone alternatives. We propose it as a variant of the regret theory developed by Bell and Loomes and Sugden (referred to as RT-B/LS in this paper) for economic decisions under uncertainty. RT-B/LS assumes that regret depends simply on the differences of the utilities of the compared alternatives. In this paper we show that this difference between RDRM and RT-B/LS has verifiable implications for choosing the "best" from a set of deterministic alternatives. Unlike the classical EUT, the RT$\mathrm{B} / \mathrm{LS}$ and RDRM utilities are specified in the context of all the alternatives under consideration and are coupled through the anticipated regret that an individual experiences when intuitively or instinctively comparing alternatives. We propose CURA as an extension of Cost-Utility Analysis (CUA) whereby each alternative is characterized in terms of its monetary cost, an aggregate performance/effectiveness utility, and an anticipated regret value. The model can readily be extended to incorporate rejoicing to suit decision makers who seek it. CURA addresses the needs of the DM who desires reasonably detailed information and visibility into the alternatives rather than a single number.

The remainder of the paper is organized as follows. In Section 2 we illustrate the limitations of the classical linear additive utility model and the need for a realistic MAUA model that incorporates rational emotions such as regret. In Section 3 we develop RDRM and investigate its properties. In Section 4 we propose CURA as a desirable MCDA method. Section 5 demonstrates the application of the proposed approach using a realistic trade-off study. 
Concluding remarks are presented in Section 6. Appendix A contains proofs of some properties of rank reversal in the RDRM. An example of rank reversal in AHP that reasonable people consider irrational is included in Appendix B.

\section{A REVIEW OF CHOICE REGRET MODELS}

In this section we briefly examine the minimax loss criterion proposed by Savage [1951] and the regret theory model of Bell and Loomes and Sugden (RT-B/LS). We begin by showing that the Conventional Linearly Additive Utility (CLAU) model cannot accommodate regret and therefore it has limited predictive descriptor capabilities. (We use the qualifier "conventional" to differentiate between the linear additive model based on classical utility theory and those based on the recent developments mentioned in the Introduction.) We illustrate these models using a simple selection problem that consists of three alternatives $\left\{A_{1}, A_{2}, A_{3}\right\}$ with two criteria $\left\{C_{1}\right.$, $\left.\mathrm{C}_{2}\right\}$ as specified by Example 1 in Table I. (It is adapted from Example 8.2 of Pomerol and Barba-Romero [2000: 208].)

Table I. Example 1 data

\begin{tabular}{|c|c|c|}
\cline { 2 - 3 } \multicolumn{1}{c|}{} & \multicolumn{2}{c|}{ Utilities } \\
\hline Alternatives & \multicolumn{1}{c|}{$\mathbf{C}_{1}$} & \multicolumn{1}{c|}{$\mathbf{C}_{\mathbf{2}}$} \\
\hline $\mathbf{A}_{\mathbf{1}}$ & 0.20 & 0.80 \\
$\mathbf{A}_{\mathbf{2}}$ & 0.40 & 0.40 \\
$\mathbf{A}_{3}$ & 0.80 & 0.20 \\
\hline
\end{tabular}

Notes: 1. Criteria $\mathrm{C}_{1}$ and $\mathrm{C}_{2}$ with higher utilities are preferred.

2. Several sets of weights are considered in the text.

3. To make the example concrete, the reader may wish to consider $\left\{\mathrm{A}_{1}, \mathrm{~A}_{2}, \mathrm{~A}_{3}\right\}$ to be three entertainment centers and $\left\{\mathrm{C}_{1}, \mathrm{C}_{2}\right\}$ to be image and sound.

\subsection{The Conventional Linear Additive Utility Function}

Numerous MUFs have been proposed for aggregating the individual utilities into a single scalar utility. The most widely used form is the CLAU function [Daniels, Werner, and Bahill, 2001]. It is valid if and only if the criteria are mutually preferentially independent [Keeney and Raiffa, 1976]. Consider Example 1 in Table I. Within the CLAU model, the utility of alternative $A_{i}(i=$ $1,2,3)$ is given by $U\left(A_{i}\right)=w_{1} * U_{1}\left(A_{i}\right)+\left(1-w_{1}\right) * U_{2}\left(A_{i}\right) \equiv w_{1} * u_{i 1}+\left(1-w_{1}\right) * u_{i 2}$. It is easy to verify that there are no weights with values $0 \leq \mathrm{w}_{1} \leq 1$ for which $A_{2}$ has higher utility than both $A_{1}$ and $A_{3}$. The individual who bases his choice on the above model would choose either $A_{1}$ or $A_{3}$ even though they have the lowest utilities for criteria $C_{1}$ and $C_{2}$, respectively. When faced with this choice many individuals would choose $\mathrm{A}_{2}$ in contradiction with the CLAU model. Some proponents of MAUA might argue that these people have misapplied the model or are confused about the assessment of their utilities. We think that this argument addresses only a subset of the problems and is therefore of limited validity. The CLAU model is a compensatory model and the poor performance of any criterion can be compensated by the other criteria. We 
concur that it should not be used when this property is not desired; "musts" need to be treated separately [Kepner and Tregoe, 1965]. However, as we discuss in the Introduction, there are important considerations such as emotions that affect many decisions and conventional MAUA does not address adequately and/or ignore. The simple Example 1 illustrates that the CLAU model needs refinements to make it a better descriptive predictor or aid for MCDA. Even Markowitz [1997] who on page 208 identifies himself as a proponent of classical EUT on page 209 questions its applicability to the making of decisions in which emotions are important influences because he recognizes the following as a serious shortcoming:

"The modern notion of utility avoids any hedonistic interpretation of the utility curve."

\subsection{Minimax Regret}

Minimax regret is a strategy for decision making under uncertainty whereby the DM chooses the alternative with the minimum worst possible outcome in order to minimize regret. It requires knowledge only of the possible outcomes and not their probabilities. It hypothesizes that a DM who chooses an alternative $A_{i}$ experiences a level of regret for state of nature $S_{k}$ equal to the difference between its best possible value and its actual value for $A_{i}, R_{i k}=\operatorname{Max}_{j}\left(u_{j k}\right)-u_{i k}$. The minimax regret decision rule is to choose the alternative $A_{i}$ with the minimum value of $R_{i k}$. By identifying the states of nature with the criteria of the alternatives, minimax regret is directly applicable to MCDA under certainty. Consider Example 1. The associated regret matrix is given in Table II. As expected, $\mathrm{A}_{2}$ is the recommended choice because it is the alternative with the minimum of the maximum regret values. Depending on the context, this may be a more desirable choice than $A_{1}$ or $A_{3}$, that as seen in Section 2.1, are the only possible highest utility choices of the CLAU model.

Table II. Minimax regret matrix for Example 1

\begin{tabular}{|c|r|r|r|}
\cline { 2 - 4 } \multicolumn{1}{c|}{} & \multicolumn{3}{c|}{ Regret values } \\
\hline Alternatives & \multicolumn{1}{c|}{$\mathbf{C}_{1}$} & \multicolumn{1}{c|}{$\mathbf{C}_{2}$} & Maximum \\
\hline $\mathbf{A}_{1}$ & 0.60 & 0.00 & 0.60 \\
$\mathbf{A}_{2}$ & 0.40 & 0.40 & 0.40 \\
$\mathbf{A}_{3}$ & 0.00 & 0.60 & 0.60 \\
\hline
\end{tabular}

Because minimax regret is driven solely by regret, it lends itself to irrational choices. For example, a small disadvantage in a single criterion regardless of its importance can eliminate alternatives with criteria that are both superior and of greater relative importance. Given the undesirable properties of minimax regret, the notion of regret had been largely dismissed in decision analysis until the development of RT-B/LS. As we discuss in the following section, RT-B/LS does not minimize the maximum regret; instead it trades-off regret against the classical utilities to identify a decision that balances benefits with regret in accordance with the DM's 
preferences.

\subsection{The Regret Theory of Bell and Loomes and Sugden (RT-B/LS)}

RT-B/LS incorporates the concepts of (1) regret and its counterpart, rejoicing, and (2) disappointment and its counterpart, elation, into EUT by means of a modified utility function that is the sum of the utilities for performance and psychological contributions. Regret-rejoicing and disappointment-elation are psychological experiences that play important parts under different situations. Disappointment and elation depend on the risk and opportunity of the selected action under a state of uncertainty [Browning and Hillson, 2004]. A rational individual feels some level of disappointment in a decision making under uncertainty when the outcome does not match up to expectations and he experiences elation when the outcome exceeds expectation. Anticipated disappointment and elation are not considerations or influences for deterministic choices. In contrast, a rational individual may experience regret and rejoicing when making decisions under certainty as well as uncertainty. Since in this paper we focus on decision making under certainty we do not consider disappointment-elation.

Consider two deterministic alternatives $A_{i}$ and $A_{j}$ with classical utilities $u_{i k}$ and $u_{j k}$, i.e. evaluated independent of the other alternatives. Assume that $A_{i}$ has a higher total utility than $A_{j}$ but that it performs worse for some criteria $C_{k}, u_{i k}<u_{j k}$. The individual who chooses $A_{i}$ may then experience regret because the performance of $C_{k}$ is worse than for the forgone alternative $\mathrm{A}_{\mathrm{j}}$. RT-B/LS assumes that the levels of regret and rejoicing depend only on the difference between the utility of "what is" and the utility of "what might have been" and not the absolute values. To simplify the presentation and because experimental studies confirm that for most individuals regret has the greater impact [Mellers, 2000] we also omit rejoicing. We note that rejoicing can be treated in an analogous manner to regret and considered as an element of the selection process by DMs who value rejoicing as well as regret. The RT-B/LS model devoid of disappointment-elation and rejoicing reduces to the following functional form,

$$
\mathrm{R}\left(\mathrm{u}_{\mathrm{ik}}, \mathrm{u}_{\mathrm{jk}}\right)=\left\{\begin{array}{l}
\mathrm{R}\left(\mathrm{u}_{\mathrm{j} k}-\mathrm{u}_{\mathrm{ik}}\right), \mathrm{u}_{\mathrm{ik}}<\mathrm{u}_{\mathrm{jk}} \\
0, \text { otherwise }
\end{array}\right.
$$

where $u_{i k}$ and $u_{j k}$ are the classical utilities. Since the level of regret should increase as the difference between compared utilities increases, $\mathrm{R}(\cdot)$ is a non-decreasing function. RT-B/LS further assumes that $\mathrm{R}(\cdot)$ is convex. (We revisit this assumption in Section 3 where we show that it has problematic implications for MCDA.) Using Eq. (1) the RT-B/LS utility of criterion $\mathrm{C}_{k}$ for $A_{i}$ in the presence of $A_{j}$ is given by

$$
U_{i k}^{j}=\left\{\begin{array}{l}
u_{i k}-R\left(u_{j k}-u_{i k}\right), u_{i k}<u_{j k} \\
u_{i k}, \text { otherwise. }
\end{array}\right.
$$

The corresponding utility for $A_{j}$ is simply obtained by interchanging the indices $i$ and $j$. For the individual who experiences no regret, Eq. (2) reduces to the classical utility. In contrast to classical utility theory, the utility of each alternative now depends on the other alternative. 
RT-B/LS extends Eq. (2) in a natural manner to a set $\mathrm{S}$ of $\mathrm{n}(\geq 2)$ alternatives with $\mathrm{m}(\geq 2)$ criteria. For every criterion $C_{k}$, a given alternative $A_{i}$ may have multiple contributions to regret that arise from comparing it with the other alternatives. The RT-B/LS MUF averages Eq. (2) over all other (n-1) alternatives and weighs it over all of the $m$ criteria:

$$
U_{i}^{S}=\sum_{k=1}^{m} \mathrm{w}_{\mathrm{k}} *\left[\mathrm{u}_{\mathrm{ik}}-\left(\frac{1}{n-1}\right) * \sum_{j=1}^{n} \mathrm{R}\left(\mathrm{u}_{\mathrm{jk}}-\mathrm{u}_{\mathrm{ik}}\right)\right] .
$$

The first term is the classical utility that measures a person's preference of each alternative independent of the other alternatives. The second term accounts for her anticipated regret over all the alternatives that she might have chosen. It compares each alternative with the other (n-1) alternatives. Following conventional MAUA, a DM now chooses the alternative with the highest total RT-B/LS utility. The choice may differ from and be reasonably preferred to the alternative with the highest CLAU because it may be a more balanced choice since it reflects rational psychological influences such as regret and rejoicing.

We now revisit Example 1 within the framework of the RT-B/LS model in Eq. (3). To proceed with a quantitative illustration we assume (1) weights $\mathrm{w}_{1}=\mathrm{w}_{2}=0.5$, and (2) a convex regret function with the values in Table III.

Table III. RT-B/LS regret values for Example 1

\begin{tabular}{|c|c|c|c|c|}
\hline $\mathbf{x}$ & 0.00 & 0.20 & 0.40 & 0.60 \\
\hline $\mathbf{R}(\mathbf{x})$ & 0.00 & 0.01 & 0.17 & 0.83 \\
\hline
\end{tabular}

Note: These values correspond to a convex function.

Using Eq. (3) and the specified regret values, one finds that the RT-B/LS utilities for alternatives $\mathrm{A}_{1}, \mathrm{~A}_{2}$, and $\mathrm{A}_{3}$ are $\mathrm{U}_{1}^{3}=0.29, \mathrm{U}_{2}^{3}=0.32$, and $\mathrm{U}_{3}^{3}=0.29$, respectively. The ranking is now in agreement with our hypothetical DM's preferences, $A_{2} \succ A_{1} \sim A_{3}$; i.e. $A_{2}$ is preferred to $A_{1}$ and $A_{3}$ and there is indifference between $A_{1}$ and $A_{3}$. This illustrates that RT-B/LS enhances the CLAU model. However, in Section 3 we show that RT-B/LS exhibits intransitivity under pairwise comparisons and inconsistencies with some empirical evidence. To resolve these problems, we formulate RDRM as a model of regret that depends on the absolutes values of the utilities of the compared alternatives.

\section{A REFERENCE-DEPENDENT REGRET MODEL (RDRM)}

\subsection{The Role of Anticipated Regret in MCDA}

In the previous two sections we showed that deterministic MCDA requires a model that explicitly incorporates psychological reactions such as anticipated regret. To proceed we explicitly define our notion of regret for decision-making under certainty by tailoring Bell's 
[1982: 961] notion of anticipated regret for decision-making under uncertainty as follows. In the process of choosing a deterministic alternative, a rational individual may decide to trade-off some benefits and forgo the alternative with the highest total value for a more balanced alternative in order to reduce his level of anticipated regret. This behavior is in conflict with conventional MAUA and EUT. As discussed in the Introduction, we consider it to be rational because most individuals value emotions as an important decision consideration along with cost and performance. Before making a final decision, a good DM revisits the preferred solutions, analyzes potential problems, and evaluates disadvantages to ensure an acceptable level of regret. There are times when her choice may be more justifiable and hence more rationale than the highest-utility alternative identified by today's typical MAUA.

To motivate the need for modifications to RT-B/LS, consider the following two situations:

- Case 1: Car A is rated "very high reliability" with classical utility of 1.0 out of a possible 1.0. Car B is rated "high reliability" with a classical utility of 0.8 .

For the other criteria the preference is Car A $\succ$ Car B.

- Case 2: Car C is rated "average reliability" with a classical utility of 0.6 out of a possible 1.0. Car D is rated "below average reliability" with a classical utility of 0.4 . For the other criteria the preference is Car $\mathrm{C} \succ$ Car D.

One can imagine a person who feels worse in Case 2 than in Case 1 for having chosen the car with the lower reliability. This behavior is inconsistent with RT-B/LS that predicts identical levels of regret for Cases 1 and 2 because the differences of the utilities of the compared reliabilities both equal 0.2 . More generally, real-world experience suggests that a person's level of regret when he chooses a multi-attribute alternative often depends explicitly on the absolute values of the utilities of the chosen and forgone alternatives rather than simply the differences. We propose RDRM to account this behavior. The RDRM then explains that the levels of regret experienced in Cases 1 and 2 are different because of the different classical utilities, $(0.8$ and 1.0) vs. $(0.4$ and 0.6$)$. The RDRM has similarities to prospect theory, which evaluates utility of monetary gains and losses with respect to a reference point. We now proceed to develop RDRM, investigate some of its properties, and compare it in detail with RT-B/LS.

\subsection{Development of RDRM}

The RDRM assumes a regret function of the following functional form:

$$
\mathrm{R}\left(\mathrm{u}_{\mathrm{ik}}, \mathrm{u}_{\mathrm{jk}}\right)=\left\{\begin{array}{l}
\mathrm{G}\left(1-\mathrm{u}_{\mathrm{ik}}\right)-\mathrm{G}\left(1-\mathrm{u}_{\mathrm{j} k}\right), \mathrm{u}_{\mathrm{ik}}<\mathrm{u}_{\mathrm{jk}} \\
0, \text { otherwise }
\end{array}\right.
$$

where the utilities range between 0 and 1 . We refer to $\mathrm{G}(\cdot)$ as the regret-building function. It measures the level of regret referenced to the maximum possible utility normalized to 1 . Since the level of regret increases as the difference between the compared utilities increases, $G(\cdot)$ is a non-decreasing function. For a non-linear form of $\mathrm{G}(\cdot)$ a person's regret level depends explicitly 
on the utilities of the "what is" and the "what might have been" alternatives and not simply the differences. Given the lack of data, a more general or complex dependence does not seem warranted at the present time. In general, each criterion $\mathrm{C}_{\mathrm{k}}$ may be associated with a different regret-building function $G_{k}(\cdot)$. In this paper we assume a common function for all criteria under consideration. The individual who chooses alternative $A_{i}$ from a set $S$ of $n(\geq 2)$ alternatives with $\mathrm{m}$ criteria is assumed to experience a total level of regret given by

$$
R_{i}^{S}=\left(\frac{1}{n-1}\right)^{*} \sum_{j=1}^{n} \sum_{k=1}^{m} \mathrm{w}_{\mathrm{k}}^{*} \max \left(\mathrm{G}\left(1-\mathrm{u}_{\mathrm{ik}}\right)-\mathrm{G}\left(1-\mathrm{u}_{\mathrm{jk}}\right), 0\right)
$$

\subsection{Selection of a Regret-Building Function}

Our objective is to select a simple and studied function $G(\cdot)$ that is flexible enough to realistically represent the wide range of levels of regret experienced in MCDA by individuals depending on their emotions, context, and alternatives. There is no fundamental law of nature that requires this function to be concave or convex. Even though RT-B/LS assumes a convex regret function, Loomes and Sugden [1982: 810] state:

"On the face of it there seems to be no a priori reason for preferring any one of the assumptions to the others. They are simply alternative assumptions about human psychology and a choice between them should be made mainly on the basis of empirical evidence."

In fact we think that it is very likely that a person may be regret adverse in one range and not in another depending on the utilities of the compared alternatives.

The Wymore [1993] scoring function SSF1 meets our requirements. It is given by

$$
G(x)=\left\{\begin{array}{l}
\frac{1}{1+(B / x)^{2 * S^{*}(B+x)}}, x>0 \\
0, \text { otherwise. }
\end{array}\right.
$$

$\mathrm{G}(\cdot)$ ranges between 0 and $1: \mathrm{G}(0)=0, \mathrm{G}(\mathrm{B})=0.5$, and $\mathrm{G}(\infty)=1$. The two parameters $\mathrm{B}$ and $\mathrm{S}$ characterize the shape. The larger the value of $S$, the steeper $G(\cdot)$ is and the faster it approaches 1. The curve is convex for values below B and concave above it resulting in a shape that flattens both near 0 and 1 . We find this to be intuitively consistent with the levels of regret experienced by individuals. It is interesting to note that it captures the behavior of the value function in prospect theory formulated by Kahneman and Tversky [1979]. The parameters B and S can be determined from regret data, when available, by setting one of the two variables in Eq. (4) equal to 1 . This significantly simplifies the analysis compared to a more general function of two variables. 
Figure 1 illustrates the flexibility and properties of $\mathrm{G}(\cdot)$ for several values of B and $\mathrm{S}$.

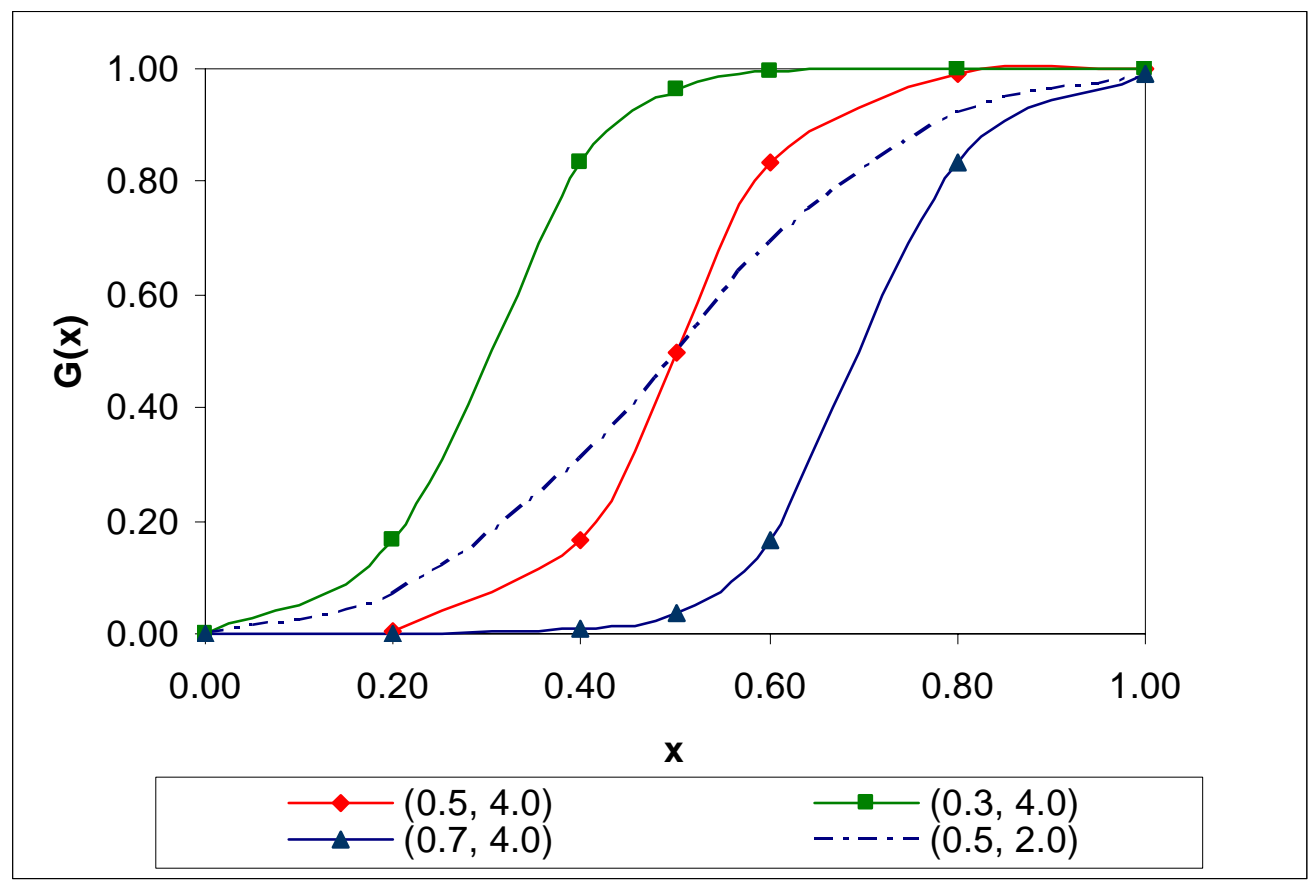

Figure 1. Curves corresponding to the Wymore scoring function with several values of $\mathrm{S}$ and $\mathrm{B}$. The curves are labeled by $(\mathrm{B}, \mathrm{S})$. The curve $(0.5,4.0)$ over the range 0.0 to 0.6 corresponds to the regret values in Table III.

In a real-world application the analyst would determine these parameters by querying the DM about the levels of regret that he experiences for each criterion. The RDRM function given by Eq. (4) cannot be drawn as a single curve because the level of regret depends explicitly on the compared utilities. For a given set of parameters $(\mathrm{B}, \mathrm{S})$ the RDRM function represents a family of curves where each curve corresponds to a reference or chosen alternative with a different utility for the compared criterion. Figure 2 shows regret effects for several different comparisons. The data assumes a reference parameter $\mathrm{B}=0.5$, which we feel is appropriate for a utility scale with the following ratings [Lootsma, 1997: 83]: $0.90-1.0$ for excellent, $0.89-0.70$ for very good to good, $0.69-0.50$ for fair/good to fair, 0.49 - 0.4 for fair/poor, 0.39 - 0 for poor. The level of regret experienced by an individual depends on the absolute values of the criteria of the compared alternatives. A chosen alternative with a criterion utility $<0.5$ is judged as very painful when there is another alternative with a corresponding criterion utility $>0.5$. This is consistent with the experimental studies of emotions including anticipated regret reported by Mellers [2000]. The individual who heavily weighs regret when selecting an alternative with one or more criteria judged to be less than "good" may then instead select an alternative with all criteria judged to be at a minimum "good" rather than the alternative with the highest classical utility. 


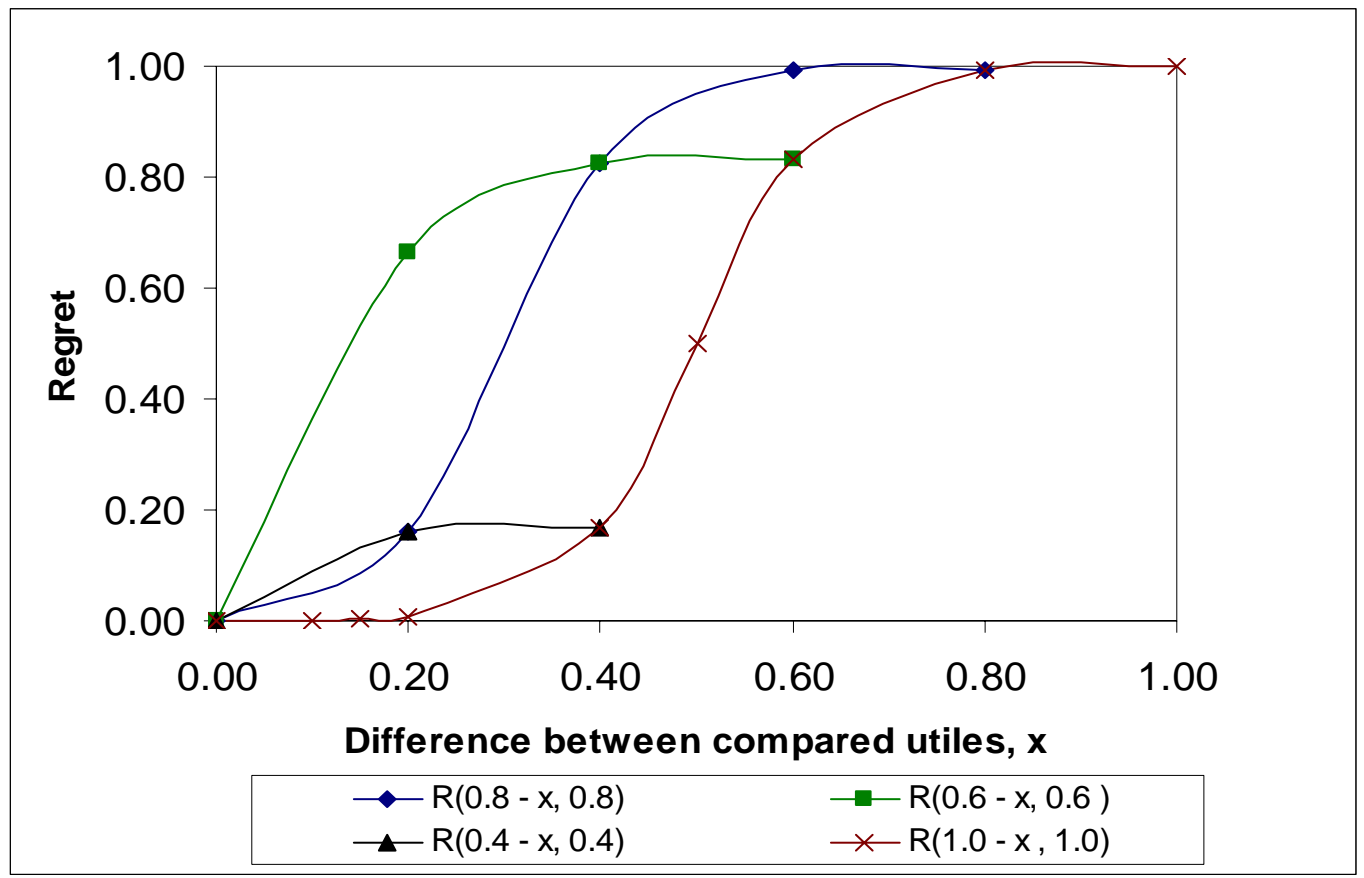

Figure 2. Family of RDRM regret functions given by Eq. (4) using the Wymore scoring function SSF1 with parameters $\mathrm{B}=0.5$ and $\mathrm{S}=4.0$. The curves correspond to reference or chosen alternatives with utilities for the compared criterion of $1.0,0.8,0.6$, and 0.4 , respectively.

\subsection{Some Implications of RDRM}

\subsubsection{Reference-Dependence}

RDRM predicts different levels of regrets for the two motivational cases introduced in Section 3.1. Consider Figure 2. The data points $R(0.8,1.0)$ and $R(0.4,0.6)$ correspond to levels of regret of 0.01 and 0.67 for Cases 1 and 2, respectively. This is in contrast with RT-B/LS which predicts equal levels of regret for both cases given the equal utility differences of 0.2 . Different individuals experience different levels of regret and these would be modeled with different parameters $\mathrm{B}$ and $\mathrm{S}$.

There are recent experimental investigations of preference reversal that indicate that the experienced level of regret is reference-dependent. Based on their investigations, Cubitt et al. [2004: 709] conclude:

"We find a pattern of preference reversals that is inconsistent with all of the best-known explanations of the phenomenon proposed by economists, with the fundamental economic assumption of context-free preferences, and with several psychological theories of preference reversal."

We consider this as additional evidence in support of RDRM. 


\subsubsection{Intransitivity}

Consider the three alternatives with three criteria specified by the decision matrix in Table IV.

Table IV. Example 2. An example of intransitivity in RT-B/LS

\begin{tabular}{|c|c|c|c|}
\cline { 2 - 4 } \multicolumn{1}{c|}{} & \multicolumn{3}{c|}{ Criteria, Utilities } \\
\hline Alternatives & $\mathbf{C}_{1}$ & $\mathbf{C}_{2}$ & $\mathbf{C}_{3}$ \\
\hline $\mathbf{A}_{1}$ & $\mathrm{Z}$ & $\mathrm{y}$ & $\mathrm{x}$ \\
$\mathbf{A}_{2}$ & $\mathrm{X}$ & $\mathrm{Z}$ & $\mathrm{y}$ \\
$\mathbf{A}_{3}$ & $\mathrm{y}$ & $\mathrm{x}$ & $\mathrm{Z}$ \\
\hline Weights & $1 / 3$ & $1 / 3$ & $1 / 3$ \\
\hline
\end{tabular}

Note: With no loss of generality we assume utilities $\mathrm{x}>\mathrm{y}>\mathrm{z}$.

This is one of several examples that have been presented in the literature to illustrate that RTB/LS exhibits intransitivity [Starmer, 2000: 356]. Based on the symmetry of Example 2 we expect the three alternatives to be equivalent and a rational individual to be indifferent to any one of the three choices. However, as we show below, pairwise comparisons within the framework of RT-B/LS lead to cyclic preferences but not within the framework of RDRM.

\subsubsection{RT-B/LS Analysis}

We follow the analysis given by Starmer [2000: 356]. Consider Table IV and the preference between $A_{1}$ and $A_{2}$ within the context of the pair $\left\{A_{1}, A_{2}\right\}$. We compute the associated utilities using Eq. (3):

$$
\begin{aligned}
& \mathrm{U}^{2}=1 / 3^{*}(\mathrm{x}+\mathrm{y}+\mathrm{z})-1 / 3^{*} \mathrm{R}(\mathrm{x}-\mathrm{z}) \\
& \mathrm{U}^{2}{ }_{2}=1 / 3^{*}(\mathrm{x}+\mathrm{y}+\mathrm{z})-1 / 3^{*}[\mathrm{R}(\mathrm{x}-\mathrm{y})+\mathrm{R}(\mathrm{y}-\mathrm{z})] .
\end{aligned}
$$

Combining the above expressions yields

$$
\mathrm{U}^{2}{ }_{1}-\mathrm{U}^{2}{ }_{2}=(1 / 3) *[-\mathrm{R}(\mathrm{x}-\mathrm{z})+\mathrm{R}(\mathrm{x}-\mathrm{y})+\mathrm{R}(\mathrm{y}-\mathrm{z})] .
$$

An additional assumption of RT-B/LS is that $R(\cdot)$ is convex; i.e. $R(x-z)>R(x-y)+R(y-z)$. This implies that the DM should prefer $A_{2} \succ A_{1}$. Applying the same reasoning to the two other pairs, one finds $A_{3} \succ A_{2}$ and $A_{1} \succ A_{3}$. The net result is cyclic preferences $A_{2} \succ A_{1}, A_{3} \succ A_{2}$, and $A_{1} \succ A_{3}$.

To show that this irrational ranking is associated with the process of pairwise comparisons, we directly compute the utilities of the alternatives for the set $\left\{A_{1}, A_{2}, A_{3}\right\}$ using Eq. (3). One finds that the three alternatives have equal RT-B/LS total utilities,

$$
\mathrm{U}^{3}{ }_{1}=\mathrm{U}^{3}{ }_{2}=\mathrm{U}^{3}{ }_{3}=1 / 3^{*}(\mathrm{x}+\mathrm{y}+\mathrm{z})-1 / 6^{*}[\mathrm{R}(\mathrm{x}-\mathrm{z})+\mathrm{R}(\mathrm{x}-\mathrm{y})+\mathrm{R}(\mathrm{y}-\mathrm{z})]
$$


The use of a MUF assigns a unique cardinal utility to each alternative and thereby eliminates intransitivity as a possibility.

To make the example more concrete, we quantify it as follows: $\mathrm{x}=0.6, \mathrm{y}=0.4, \mathrm{z}=0.2$, and the RT-B/LS regret function in table III. The preference for the pair $\left\{\mathrm{A}_{1}, \mathrm{~A}_{2}\right\}$ is given by

$$
\mathrm{P}\left(\mathrm{A}_{1}, \mathrm{~A}_{2}\right) \equiv \mathrm{U}_{1}^{2}-\mathrm{U}_{2}^{2}=(1 / 3) *[-\mathrm{R}(0.4)+\mathrm{R}(0.2)+\mathrm{R}(0.2)]=-0.056
$$

This implies $\mathrm{A}_{2} \succ \mathrm{A}_{1}$. Similarly, $\mathrm{P}\left(\mathrm{A}_{2}, \mathrm{~A}_{3}\right)=-0.056$ implies $\mathrm{A}_{3} \succ \mathrm{A}_{2}$ and $\mathrm{P}\left(\mathrm{A}_{1}, \mathrm{~A}_{3}\right)=0.056$ implies $A_{1} \succ A_{3}$. This quantitatively illustrates intransitivity within RT-B/LS. Saari [1999] illustrates the phenomenon of cyclic preferences by the interesting analogy of three equal forces acting on a point body along three planar directions with $120^{\circ}$ symmetry. When one considers the three forces as an ensemble, their equivalence is clear based on the symmetry. However, if one of the forces is removed the symmetry is broken and it is no longer evident that the forces have equal magnitudes.

\subsubsection{RDRM Analysis}

We now pairwise compare the three alternatives using the RDRM regret values given by Eq. (5). The level of regrets experienced for choices $A_{1}$ and $A_{2}$ in the context of the pair $\left\{A_{1}, A_{2}\right\}$ are

$$
\begin{aligned}
\mathrm{R}^{2}{ }_{1} & =\mathrm{R}(\mathrm{z}, \mathrm{x})=(1 / 3) *[\mathrm{G}(1-\mathrm{x})-\mathrm{G}(1-\mathrm{z})], \\
\mathrm{R}^{2}{ }_{2} & =\mathrm{R}(\mathrm{z}, \mathrm{y})+\mathrm{R}(\mathrm{y}, \mathrm{x}) \\
& =(1 / 3)^{*}[\mathrm{G}(1-\mathrm{y})-\mathrm{G}(1-\mathrm{z})]+(1 / 3) *[\mathrm{G}(1-\mathrm{x})-\mathrm{G}(1-\mathrm{y})] \\
& =(1 / 3)^{*}[\mathrm{G}(1-\mathrm{x})-\mathrm{G}(1-\mathrm{z})] .
\end{aligned}
$$

The two RDRM regret levels are equal. Following RT-B/LS, we assume that the RDRM utilities are obtained from the classical utilities by subtracting the RDRM regret values: $\mathrm{U}^{* 2}{ }_{1}=\mathrm{U}_{1}-\mathrm{R}^{2}{ }_{1}$ and $U^{* 2}{ }_{2}=U_{2}-R^{2}{ }_{1}$. The equivalence between $A_{1}$ and $A_{2}$ is preserved. Similarly, it follows that $\mathrm{A}_{2} \sim \mathrm{A}_{3}$ and $\mathrm{A}_{1} \sim \mathrm{A}_{3}$. For Example 2, RDRM with a common regret function for the three criteria preserves the equivalence of the three alternatives under pairwise comparisons. In Section 3.4.3 we show that RDRM ensures the transitive pairwise rankings of three alternatives with any number of criteria $\geq 3$ because it satisfies the special property of additive transitivity; i.e. $R(z, x)=R(z, y)+R(y, x)$.

\subsubsection{Intransitive Conjoint Measurements}

There has been much interest over the past $40+$ years in intransitive preferences and a number of mathematical representations, known as intransitive conjoint measurements, have been proposed [Fishburn, 1991; Bouyssou and Pirlot, 1999]. The most general form is

$$
A_{i} \succeq A_{j} \Leftrightarrow P\left(A_{i}, A_{j}\right) \equiv P\left(p_{1}\left(u_{i 1}, u_{j 1}\right), p_{2}\left(u_{i 2}, u_{j 2}\right), \ldots, p_{m}\left(u_{i m}, u_{j m}\right)\right) \geq 0, \quad \text { Eq. }(10)
$$


where the $p_{\mathrm{k}}$ are real-valued functions of two variables and $\mathrm{P}(\cdot)$ is a non-decreasing real-valued function in all its arguments. (The symbol $\Leftrightarrow$ stands for "if and only if".) Intransitive preferences $A_{i} \succ A_{j} \succ A_{k} \succ A_{i}$ are accounted for by $\mathrm{P}\left(A_{i}, A_{j}\right)>0, P\left(A_{j}, A_{k}\right)>0$, and $P\left(A_{k}, A_{i}\right)$ $>0$. Unless special properties are imposed on $\mathrm{P}(\cdot)$, it is evident that Eq. (10) can result in intransitive preferences. The functions that satisfy the additive transitivity property are the exception (see Section 3.4.3). The RDRM regret function in Eq. (4) satisfies this property and thereby ensures transitive preferences for pairwise comparisons. RT-B/LS does not because it assumes that $\mathrm{R}(\cdot)$ is convex (see Section 3.4.2.1). The Analytic Hierarchy Process (AHP) [Saaty, 2000] in both the ideal and distributive (original AHP) synthesis modes can result in intransitive preferences for pairwise comparisons [Triantaphyllou, 2000: 220].

A basic assumption of MAUA is the existence of a MUF that assigns a cardinal utility to each alternative. This avoids pairwise comparisons and ensures an unambiguous ranking for a set of three or more alternatives. In contrast, a conjoint measurement is a binary relation of preference applicable specifically to pairwise comparisons. Concerning its relevance to MCDA, Fishburn [1991: 117] states:

"When a choice is required from a set of three or more alternatives, what justifies basing it on binary preferences, even when they are transitive? This raises issues of the relationship between preference and choice, and that relationship is far from obvious..."

Friedman [1999] in a very insightful and humorous paper advocates completely dropping pairwise comparisons and using a systems approach for decision-making.

We find the use of intransitive conjoint measurements in MAUA to be disturbing for the following reasons. It is paradoxical to incorporate intransitive preferences in a model that can only generate transitive rankings. Conventional logic considers transitivity to be one of the axioms of rational individual choice. It is hard to justify intransitive conjoint measurements as necessary for providing a better MAUA model. Starmer [2000: 363], based on his review of experimental evidence, concludes:

"The bottom line is that economists do not have a theory of non-transitive behavior that is consistent with the available evidence..."

The RDRM offers a solution with the following desirable properties: (1) it incorporates more realistic and appropriate interactions among criteria and alternatives than the CLAU, AHP, and RT-B/LS models, and (2) it preserves transitivity under pairwise comparisons.

\subsubsection{Parallelism between Example 2 and a Voting Problem}

We now approach Example 2 from the perspective of a voting problem. There is value to pursuing such an approach because the voting problem has been extensively studied for over 200 years and it provides additional insight into intransitivity and cyclic preferences. Consider the decision matrix in Table IV. If we identify the three criteria with three voters $\left\{\mathrm{C}_{1}, \mathrm{C}_{2}, \mathrm{C}_{3}\right\}$, the three alternatives with three candidates $\left\{A_{1}, A_{2}, A_{3}\right\}$, and the values $x, y, z$ with the voters' 
preferences, Example 2 can be viewed as a voting problem. Table $\mathrm{V}$ is the equivalent voting preference table.

Table V. Voting preference table for 3 voters and their rankings of 3 candidates

\begin{tabular}{|c|c|c|}
\hline Voter $\mathbf{C}_{1}$ & Voter $\mathbf{C}_{\mathbf{2}}$ & Voter $\mathbf{C}_{3}$ \\
\hline $\mathrm{A}_{2}$ & $\mathrm{~A}_{3}$ & $\mathrm{~A}_{1}$ \\
$\mathrm{~A}_{3}$ & $\mathrm{~A}_{1}$ & $\mathrm{~A}_{2}$ \\
$\mathrm{~A}_{1}$ & $\mathrm{~A}_{2}$ & $\mathrm{~A}_{3}$ \\
\hline
\end{tabular}

Notes: 1. The above preference table corresponds to the decision matrix in Table IV.

2. Each voter lists the candidates in order of decreasing preference.

Each individual voter is internally consistent and satisfies transitivity. For example, voter $\mathrm{C}_{1}$ prefers $A_{2} \succ A_{3} \succ A_{1}$. (One can imagine a voting ballot on which voter $C_{1}$ could enter choices $\mathrm{A}_{2}, \mathrm{~A}_{1}, \mathrm{~A}_{2}$ (or some other equally irrational choices) for positions 1,2 , and 3 , respectively. Given such voters and voting ballots, it would not be surprising to have election results that are inconsistent with the objectives of a democratic voting system.) We now consider several voting schemes within the framework of pairwise comparisons.

Majority rule. The results of the votes are treated as follows:

- 2 out of 3 voters prefer $A_{1}$ to $A_{2}$; the majority rule ranking is $A_{1} \succ A_{2}$.

- 2 out of 3 voters prefer $A_{3}$ to $A_{1}$; the majority rule ranking is $A_{3} \succ A_{1}$.

- 2 out of 3 voters prefer $A_{2}$ to $A_{3}$; the majority rule ranking is $A_{2} \succ A_{3}$.

The above voting scheme results in cyclic preferences $A_{1} \succ A_{2}, A_{3} \succ A_{1}$, and $A_{2} \succ A_{3}$. It is a well-known result going back to Condorcet in the eighteenth century that intransitivity can emerge from group decisions even when the individuals making up the group are internally consistent. The cyclic preferences arise from the use of pairwise comparisons and ordinal utilities. This paradox and other peculiarities of ordinal voting systems have been formalized by Arrow [1951] in what is now commonly known as Arrow's Impossibly Theorem [Barrow, 1998].

Vote tallying using RT-B/LS and RDRM. Consider the RT-B/LS analysis in Section 3.4.2.1. We identify the utilities with the scores that each candidate receives in the pairwise comparisons. The outcome of the vote using the RT-B/LS utilities is cyclic preferences $A_{2} \succ A_{1}, A_{3} \succ A_{2}$, and $A_{1} \succ A_{3}$. In contrast, from the analysis in Section 3.4.2.2 it follows that assigning voting scores based on the RDRM utilities results in a tie. Both of these results can be formally derived as consequences of the following theorem due to Saari [1999: Theorem 3]:

Saari's Theorem. For any number of voters $\geq 3$ the pairwise rankings of three candidates $\left\{A_{1}, A_{2}, A_{3}\right\}$ are transitive if the differences between the pairwise tallies satisfy the relationship (additive transitivity property) $T\left(A_{1}, A_{2}\right)+T\left(A_{2}, A_{3}\right)=T\left(A_{1}, A_{3}\right)$.

To establish the relevance of Saari's theorem to Example 2, we identify $T\left(A_{1}, A_{2}\right)$ with the difference between the RDRM utilities, $T\left(A_{1}, A_{2}\right)=U^{* 2}{ }_{2}-U^{* 2}$, and similarly for the other two 
pairs. In Section 3.4.2.2 we showed that RDRM satisfies the additive transitivity property. From Saari's theorem it therefore follows that RDRM with a common regret function for all criteria ensures that the pairwise ranking of three alternatives with any number of criteria $\geq 3$ is transitive. It also follows that conjoint measurements like RT-B/LS that do not satisfy the additive transitivity property violate transitivity under pairwise comparisons.

\subsubsection{Rank Reversal}

\subsubsection{Example 1 Revisited}

We compute the RDRM regret values using Eq. (5) and the regret function given by Eq. (6) with $\mathrm{B}=0.6$ and $\mathrm{S}=4.0$ for both criteria. We note that these parameters are selected to model a level of regret that results in rank reversal. For comparison with the RT-B/LS results of Section 2.4 we define the RDRM utilities by subtracting the RDRM regret values from the classical utilities in accordance with Eq. (3). The corresponding RDRM utilities for alternatives $A_{1}, A_{2}$, and $A_{3}$ are $\mathrm{U}^{* 3}{ }_{1}=0.14, \mathrm{U}_{2}{ }_{2}=0.15$, and $\mathrm{U}_{3}{ }_{3}=0.14$. The numerical values reflect differences between RDRM and RT-B/LS; but the rankings are consistent, i.e. $\mathrm{A}_{2} \succ \mathrm{A}_{1} \sim \mathrm{A}_{3}$. To illustrate rank reversal we drop $A_{3}$. The RDRM utilities of $A_{1}$ and $A_{2}$ change to $\mathrm{U}^{* 2}{ }_{1}=0.27$ and $\mathrm{U}^{* 2}{ }_{2}=0.15$, respectively. The ranking has flipped to the preference $A_{1} \succ A_{2}$. Similarly, we drop $A_{3}$ for the RT-B/LS analysis in Section 2.4 and obtain $\mathrm{U}^{2}{ }_{1}=0.50$ and $\mathrm{U}_{2}{ }_{2}=0.32$. RDRM and RT-B/LS both exhibit rank reversal.

\subsubsection{The Rationality of Preference Reversal and Implications for Rank Reversal}

(Behavioral economists and psychologists refer to the human behavior as "preference reversal" rather than "rank reversal" to differentiate it from a possible mathematical artifact.) Laboratory studies confirm that individuals (1) exhibit preference reversal, (2) select the best alternative among the Pareto optimal ones, and (3) avoid choosing dominated alternatives even on a single criterion [Saaty, 2000: 136]. Given these notions, we interpret the rank reversal in Example 1 as a change in preference from $\left(A_{2}\right.$ given $A_{1}$ and $\left.A_{3}\right) \succ\left(A_{1}\right.$ given $A_{2}$ and $\left.A_{3}\right)$ to $\left(A_{1}\right.$ given $\left.A_{2}\right) \succ$ $\left(A_{2}\right.$ given $\left.A_{1}\right)$. Since $A_{3}$ is dominant under $C_{1}$, dropping it changes the situation and an individual may rationally perceive the performance of $\mathrm{A}_{1}$ for criterion $\mathrm{C}_{1}$ to be significantly inferior in the presence of $\mathrm{A}_{3}$ than in its absence. With this interpretation there is no violation of transitivity. Because we have modeled regret, rank reversal appears as the consequence of a rational choice associated with a dynamic situation or two distinct situations. When dealing with discrete choices under certainty, most rational individuals and decision theorists and practitioners are inclined to agree that valuation should not be separated from context. We then deem reasonable that any credible MCDA model for both the normative and descriptive perspectives should satisfy the following three verifiable properties:

Property 1 (Independence of dominated alternatives). Consider two alternatives with ranking $A_{i} \succ A_{j}$. Adding an alternative that is dominated by $A_{i}$ or dropping an alternative that is dominated by $A_{j}$ should preserve the ranking $A_{i} \succ A_{j}$.

Property 2 (No imposed rank reversal). The occurrence of rank reversal or rank preservation following adding or dropping an alternative should reflect an individual's preference in response 
to rational emotions such as anticipated regret.

Property 3 (Negative side effects associated with inferior substitutions). Replacing an alternative by one that it dominates may change the relative rankings of the other alternatives.

Properties 1 and 2 are intuitively reasonable and we consider a rank reversal that violates them to be irrational or an abnormality. Property 1 is consistent with Luce and Raiffa's [1958: 288] Axiom 6 and Axiom 7 for decision making under uncertainty. It is important to note that that Arrow's Condition 3 (Independence of irrelevant alternatives) is much more restrictive. It requires that the ordering of any subset of alternatives is not altered by changes not in this subset. Property 1 applies to situations where dominated alternatives are added and/or dropped; it does not apply to adding or dropping non-dominated or Pareto optimal alternatives. Numerous authors have refuted Arrow's Condition 3 and identified it as a source of logical contradiction [Luce and Raiffa, 1958: 338]. Property 2 requires that the MCDA model explicitly includes rational emotions such as regret as an element of decision making.

In Appendix A we prove that RDRM and RT-B/LS satisfy Properties 1 and 2. However, some popular MCDA methods violate one or both of these. Rank reversal in the AHP has been a hotly contested subject for almost twenty years [Dyer, 1990a and 1990b; Harker and Vargas, 1990; Saaty, 1990 and 2000; Triantaphyllou, 2000]. We consider rank reversal in AHP to be a mathematical artifact rather than a model of true preference reversal in response to emotions such as anticipated regret because it does not satisfy Property 1 and Property 2. For completeness, in Appendix B we reproduce an example of AHP rank reversal that most people would consider to be irrational [Dyer, 1990a]. The wide world of sports offers interesting examples of different ranking systems. The 2002 Winter Olympics woman's figure skating provided an exciting example of how rank reversal can occur in a scoring system and the impossibility of developing one that all participants and spectators would find satisfactory [Kujawski, 2003]. The CLAU model is in conflict with Property 2 because it leaves no room for rational emotions such as regret.

Property 3 is somewhat counterintuitive because it allows hurting one alternative by reducing the performance of another one. This cannot be considered desirable because it lends itself to manipulative practices. However, it is justified by empirical studies and the real-world evidence that some individuals avoid choosing dominated alternatives even on a single criterion. (We speculate that given a credible MCDA model with Properties 1 and 2, Property 3 may be necessary for mathematical or logical consistency.) To illustrate Property 3 we consider three alternatives $A_{i}, A_{j}$, and $A_{k}$ related as follows: (1) $A_{i} \succ A_{j}$, and (2) classical utilities $u_{j 1}<u_{i 1}, u_{j 1}<$ $\mathrm{u}_{\mathrm{k} 1}$, and $\mathrm{u}_{\mathrm{k} 1}<\mathrm{u}_{\mathrm{i} 1}$. Preference reversal may occur when $A_{\mathrm{k}}$ is replaced by an inferior alternative $\mathrm{A}_{\mathrm{k}}{ }_{\mathrm{k}}$ even with the only difference $\mathrm{u}_{\mathrm{k} 1}<\mathrm{u}_{\mathrm{j} 1}$. Before the substitution an individual may forgo choosing $A_{j}$ because it is dominated by $A_{k}$ for criterion $C_{1}$. After substituting $A_{k}$ with $A_{k}^{*}, A_{j}$ is not dominated by $\mathrm{A}_{\mathrm{k}}^{*}$ for criterion $\mathrm{C}_{1}$. This individual may then experience a reduced level of regret choosing $A_{j}$ and choose it rather than $A_{i}$. The situation is illustrated by an example in Tables VIa and VIb. 
Table VIa. Initial situation for illustration of Property 3

\begin{tabular}{|c|c|r|r|r|l|}
\cline { 2 - 5 } \multicolumn{1}{c|}{} & \multicolumn{2}{c|}{$\begin{array}{c}\text { Classical } \\
\text { utilities }\end{array}$} & $\begin{array}{c}\text { Regret } \\
\text { values }\end{array}$ & $\begin{array}{c}\text { Modified } \\
\text { utilities }\end{array}$ & \multirow{2}{*}{ Preference } \\
\hline Alts. & $\mathbf{C}_{1}$ & \multicolumn{1}{c|}{$\mathbf{C}_{\mathbf{2}}$} & \multicolumn{1}{c|}{$\mathbf{R}_{\mathbf{i}}$} & \multicolumn{1}{|c|}{$\mathbf{U}_{\mathbf{i}}^{3}$} & \\
\hline $\mathbf{A}_{\mathbf{1}}$ & 0.90 & 0.60 & 0.20 & 0.55 & \multirow{2}{*}{$\mathrm{A}_{1} \succ \mathrm{A}_{2}$} \\
$\mathbf{A}_{\mathbf{2}}$ & 0.65 & 0.80 & 0.25 & 0.48 & \\
$\mathbf{A}_{3}$ & 0.80 & 0.70 & --- & --- & \\
\hline Weights & 0.50 & 0.50 & \multicolumn{3}{c}{} \\
\hline
\end{tabular}

Note: Only essential data are shown.

Table VIb. After the substitution of $A_{3}$ with an inferior alternative $A_{3}{ }_{3}$

\begin{tabular}{|c|c|r|r|r|c|}
\cline { 2 - 5 } \multicolumn{1}{c|}{} & \multicolumn{2}{c|}{$\begin{array}{c}\text { Classical } \\
\text { utilities }\end{array}$} & $\begin{array}{c}\text { Regret } \\
\text { values }\end{array}$ & $\begin{array}{c}\text { Modified } \\
\text { utilities }\end{array}$ & \multirow{2}{*}{ Preference } \\
\hline Alts. & $\mathbf{C}_{1}$ & \multicolumn{1}{c|}{$\mathbf{C}_{2}$} & \multicolumn{1}{c|}{$\mathbf{R}_{\mathbf{i}}$} & \multicolumn{1}{|c|}{$\mathbf{U}^{3}{ }_{\mathbf{i}}$} & \\
\hline $\mathbf{A}_{\mathbf{1}}$ & 0.90 & 0.60 & 0.20 & 0.55 & \\
$\mathbf{A}_{2}$ & 0.65 & 0.80 & 0.15 & 0.58 & \multirow{2}{*}{$\mathrm{A}_{2} \succ \mathrm{A}_{1}$} \\
$\mathbf{A}^{*}{ }_{3}$ & 0.50 & 0.70 & ---- & --- & \\
\hline Weights & 0.50 & 0.50 & \multicolumn{2}{c}{} \\
\hline
\end{tabular}

Note that (1) $A_{3}$ is not dominated by $A_{2}$, and (2) $A^{*}{ }_{3}$ is not dominated by $A_{1}$. The noted rank reversal is therefore not in conflict with Property 1. Property 3 complements Property 1 . In Appendix A we formally prove that RDRM and RT-B/LS are consistent with Property 3.

In summary of this section, we have shown that RDRM satisfies the following properties:

1. The level of anticipated regret depends explicitly on the utilities of the "what is" and the "what might have been" alternatives and not simply the difference.

2. Regret is modeled in terms of a flexible yet simple function, a Wymore scoring function. It is convex below a reference point and concave above it. The shape depends on two parameters to be fitted to the level of regret experienced by the DM.

3. Unlike classical utility theory, the utilities of all the alternatives under consideration are interrelated.

4. Transitivity is preserved.

5. Rank reversal occurs without recourse to intransitive conjoint measurements. This reflects rank reversal as truly a rational preference reversal rather than a mathematical artifact.

6. It can readily be extended to incorporate rejoicing.

These properties are consistent with the available evidence and intuition for decision making under certainty. We think that they constitute necessary conditions for having a good descriptive and/or normative MCDA model. 


\section{COST-UTILITY-REGRET ANALYSIS}

\subsection{A Suitable Level of Detail for Trade Studies}

The standard process for complex trade-off studies, whether for systems engineering or other human endeavors, involves the following sequence of activities:

1. Structure the problem for analysis.

2. Generate solutions or identify acceptable alternatives.

3. Analyze and characterize each alternative in terms of cost, performance, effectiveness, schedule, and risk in accordance with the defined structure. For deterministic trade studies the criteria are specified by point values. When dealing with probabilistic situations or uncertainty the criteria are treated as random variables and specified using probability distribution functions.

4. Eliminate the alternatives that do not meet the technical, schedule and cost requirements.

5. Eliminate the dominated alternatives. This generally provides only limited relief because the Pareto-optimal set consists of all alternatives with at least one dominant criterion.

6. Assess the preferences of the candidate alternatives. This includes analyzing them for potential problems and robustness. (Robustness considers the impact of different approaches and models in addition to the sensitivity to the weights and utilities.)

7. Choose the "best alternative".

In this paper we focus on the last two activities, evaluation and choosing. We assume that the first five activities have been completed and that we have a set of acceptable Pareto-optimal alternatives characterized by criteria that include cost, time to delivery, performance and effectiveness measures, available resources, and others as necessary.

Most individuals find it difficult or impossible to intuitively choose a "best solution" from a set of several alternatives with more than three criteria because of one's limited ability to visualize or think in a greater than three-dimensional space [Das, 1999]. MAUA transforms the MCDA problem from the multi-dimensional space into a one-dimensional scalar function. However, this has the following serious drawbacks: (1) It hides much of the available information, and (2) any model that attempts to characterize a multi-attribute alternative by a single number requires a substantial set of assumptions. From his research of decisions under uncertainty, Shapira [1995: 51] concludes:

"Although quantities were used in discussing risk and managers tend to seek precision in estimating risk most showed little desire to reduce risk to a single quantifiable construct."

Our experience is that similar behavior holds for MCDA under certainty. Good DMs need and want adequate information and visibility into the alternatives rather than a single number when dealing with complex decisions. For many years they have realized, what is now supported by empirical evidence, that analysis and intuition are both necessary for effective decision-making [Klein, 2003]. Based on these observations we specify the following four properties for a desirable MCDA method or decision tool for deterministic trade-off studies: 
1. Provide adequate information and visibility into the alternatives rather than a single number.

2. Results presented in terms of no more than four criteria for ease of interpretation.

3. The ability to capture complex emotions such as regret and rejoicing.

4. The flexibility to trade-off classical utility in order to avoid high levels of regret or seek high levels of rejoicing.

Cost-Utility Analysis (CUA) satisfies the above first two properties [Petitti, 1994]. Under the umbrella of CUA we include cost-benefit analysis, cost-effectiveness analysis, and Cost as An Independent Variable (CAIV). In its most general form, monetary cost is kept as a distinct element and the other criteria are grouped into one or more sets of related criteria that can be aggregated using the CLAU model or other methods [Tofallis, 1999]. The MCDA problem is thereby reduced to a smaller dimensionality space with the elements being cost and a small set of aggregate utilities characterizing performance and the "ilities". DMs are no longer presented with a "best alternative" where poor performance and/or low quality may be compensated by low cost. They have visibility into the alternatives and can explicitly compare them in terms of monetary costs and a manageable set of composite utilities. Due caution is still required. DMs, designers, and analysts should heed the words of Hennesy and Patterson [1990; 70]:

"Cost/performance fallacies and pitfalls are plentiful and have ensnared many ... including ourselves."

To satisfy the other two properties we propose to treat regret and/or rejoicing as additional dimensions of the trade space and to explicitly incorporate it as an element within an extension of CUA that we refer to as Cost-Utility-Regret Analysis (CURA).

\subsection{Regret as a Dimension of the Trade Space}

Consider a set $\mathrm{S} \equiv\left\{\mathrm{A}_{\mathrm{i}}\right\}$ of $\mathrm{n}$ Pareto-optimal alternatives specified in terms of monetary cost and $\mathrm{m}$ utilities associated with the performance and effectiveness parameters. Since no alternative is absolutely better than any other one for all criteria, the individual who chooses an alternative $A_{i}$ experiences a level of anticipated regret $\mathrm{R}_{i}{ }_{i}$ that depends on the other alternatives. We propose to compute $\mathrm{R}^{\mathrm{S}}$ ising the RDRM specified by Eq. (5) and to incorporate it as an element of the trade space. Each alternative $A_{i}$ is then described by a $(m+2)$-tuple of the form $\left(C_{i}, u_{i 1}, \ldots, u_{i m}\right.$, $\mathrm{R}_{\mathrm{i}}^{\mathrm{S}}$ ). We also aggregate the utilities into a single composite performance utility using the CLAU model. Each alternative $A_{i}$ is then specified by the triplet $A_{i} \equiv\left(C_{i}, U_{i}, R_{i}^{S}\right)$ that satisfies the four properties of a desirable MCDA method specified in the previous section. When rejoicing is an important value, it can be treated in an analogous manner to regret and included as an element of the evaluation vector.

The proposed approach offers several important benefits. It avoids the difficulties, ambiguities, and pitfalls associated with aggregating the conflicting notions of cost, performance, and regret-rejoicing into a single number. Since cost, performance, and regret-rejoicing are rarely preferentially independent, the CLAU model is unlikely to be appropriate. There is no rigorous basis besides reasonable expectations for combining classical utility and regret into a single modified utility as proposed by RT-B/LS. CURA mitigates these problems in a manner 
that aids a rational indidual reach a preferred solution. Using CURA, analysts and DMs are able to compare the alternatives on the basis of cost, composite performance/effectiveness utility, and anticipated regret-rejoicing. This is highly desirable because the final decision is made by the DM using the CURA generated information rather than being the automatic result of a MCDA that provides a "best choice" based on a single number.

\section{AN ILLUSTRATIVE APPLICATION}

To demonstrate the application of the approach developed in the previous sections we consider a slightly modified version of the aircraft selection problem developed by Sen and Yang [1998: 73]. We modified the original data to better illustrate the aspects of RDRM and cost-utilityregret analysis. Our MCDA problem is defined by the data in Table VII.

Table VII. Data for the aircraft selection problem

\begin{tabular}{|c|r|r|r|r|c|c|}
\hline Alts. & $\begin{array}{c}\text { Cost } \\
\text { M\$ }\end{array}$ & $\begin{array}{c}\text { Speed } \\
\text { Mach }\end{array}$ & $\begin{array}{c}\text { Range } \\
\mathbf{1 0}^{\mathbf{3}} \mathbf{~ N M}\end{array}$ & $\begin{array}{c}\text { Payload } \\
\mathbf{1 0}^{\mathbf{3}} \mathbf{~ k g}\end{array}$ & Dependability & Maneuverability \\
\hline A1 & 6.00 & 2.0 & 1.5 & 2.0 & fair & excellent \\
A2 & 4.50 & 2.5 & 2.7 & 1.8 & poor & fair \\
A3 & 6.50 & 1.8 & 2.0 & 2.1 & good & good \\
A4 & 5.50 & 2.2 & 1.9 & 2.0 & fair & fair/good \\
\hline
\end{tabular}

Given that the focus of the paper is on the evaluation and choosing activities of MCDA we assume that the classical utilities and weights of the performance criteria have been appropriately determined as shown in Table VIIIa. We assume that for this important decision the DM values regret significantly more than rejoicing. We compute (1) the aggregated performance utility of each alternative using the CLAU function and (2) the anticipated regret level associated with the choice of each alternative using RDRM given by Eq. (5) and Eq. (6) with $\mathrm{B}=0.5$ and $\mathrm{S}=4.0$. The results are given in Tables VIIIa and VIIIb. Using these data, each alternative is characterized in terms of cost, performance utility, and regret as shown in Table IX.

Table VIIIa. Classical decision table for the aircraft selection problem

\begin{tabular}{|c|r|r|r|c|c|c|}
\cline { 2 - 6 } \multicolumn{1}{c|}{} & \multicolumn{5}{c|}{ Criteria, } & Total \\
Alts. & Speed & Range & Payload & Dep'ility & Man'ility & \\
utility \\
\hline A1 & 0.60 & 0.50 & 0.60 & 0.50 & 0.90 & 0.635 \\
A2 & 0.95 & 0.90 & 0.40 & 0.30 & 0.50 & 0.583 \\
A3 & 0.40 & 0.75 & 0.80 & 0.70 & 0.70 & 0.640 \\
A4 & 0.75 & 0.60 & 0.70 & 0.50 & 0.60 & 0.618 \\
\hline Weights & 0.25 & 0.10 & 0.10 & 0.30 & 0.25 & \multicolumn{1}{c}{} \\
\cline { 1 - 6 } & & & & & &
\end{tabular}

Note: 1. The classical total utility is computed using the CLAU function. 
Table VIIIb. RDRM regret levels for the aircraft selection problem

\begin{tabular}{|c|r|r|r|r|r|c|}
\cline { 2 - 6 } \multicolumn{1}{c|}{} & \multicolumn{4}{c|}{ Criteria, Regret values } & Total \\
\hline Alternatives & Speed & \multicolumn{1}{c|}{ Range } & \multicolumn{1}{c|}{ Payload } & Dep'ility & Man'ility & regret \\
\hline A1 & 0.11 & 0.44 & 0.10 & 0.15 & 0.00 & 0.13 \\
A2 & 0.00 & 0.00 & 0.76 & 0.62 & 0.43 & 0.37 \\
A3 & 0.77 & 0.21 & 0.00 & 0.00 & 0.06 & 0.23 \\
A4 & 0.01 & 0.11 & 0.01 & 0.46 & 0.10 & 0.18 \\
\hline
\end{tabular}

Table IX. CURA characterization of the aircraft alternatives

\begin{tabular}{|c|r|r|r|}
\hline Alternatives & \multicolumn{1}{|c|}{$\begin{array}{c}\text { Cost } \\
\text { M\$ }\end{array}$} & $\begin{array}{c}\text { Performance } \\
\text { utility }\end{array}$ & $\begin{array}{c}\text { Total } \\
\text { regret }\end{array}$ \\
\hline A1 & 6.50 & 0.635 & 0.13 \\
A2 & 4.50 & 0.583 & 0.37 \\
A3 & 6.00 & 0.640 & 0.23 \\
A4 & 5.50 & 0.618 & 0.18 \\
\hline
\end{tabular}

Consider Table IX. Each alternative is characterized by the triplet (Cost, Performance, Regret). Each alternative is Pareto-optimal with its own strong points and drawbacks. If regret were omitted, A1 would be dominated by A3 and it might be prematurely dropped as an option. Based solely on minimizing cost, the preferences are A2 $\succ \mathrm{A} 4 \succ \mathrm{A} 3 \succ \mathrm{A} 1$. Based solely on maximizing overall performance, the preferences are $\mathrm{A} 3 \succ \mathrm{A} 1 \succ \mathrm{A} 4 \succ \mathrm{A} 2$. Based solely on minimizing regret, the preferences are $\mathrm{A} 1 \succ \mathrm{A} 4 \succ \mathrm{A} 3 \succ \mathrm{A} 2 . \mathrm{A} 3$ has the best overall performance, but it causes the second highest level of regret. This high regret level is a red flag that A3 may not be a well balanced alternative. An examination of Tables VII or VIIIa reveals that A3 performs poorly on speed, an important criterion. Table IX suggests that although A1 almost ties A3 for best performance, A1 is the better balanced alternative. A2 has the lowest performance and causes the most regret, but it is still a relevant alternative because it has the lowest cost and it may be of potential interest to the DM. A4 has average values for cost and performance and a better than average value for regret. The data in Table IX can be graphically represented as shown in Figure 3. For ease of comparison, we transform cost into a cost index and regret into a regret index as follows: $\mathrm{CI} \equiv \mathrm{C}^{*} /$ Cost, $\mathrm{RI} \equiv \mathrm{R}^{*} /$ Regret, where $\mathrm{C}^{*}$ and $\mathrm{R}^{*}$ are ideal values less than the best (minimum) values of the compared alternatives. All three parameters range between 0 and 1 and higher values are preferred. What CURA achieves is to provide valuable and manageable information about each alternative to help a rational DM make a good choice. Based on this data, a rational DM may opt to trade-off some performance and/or cost benefits in order to reduce the level of anticipated regret. 


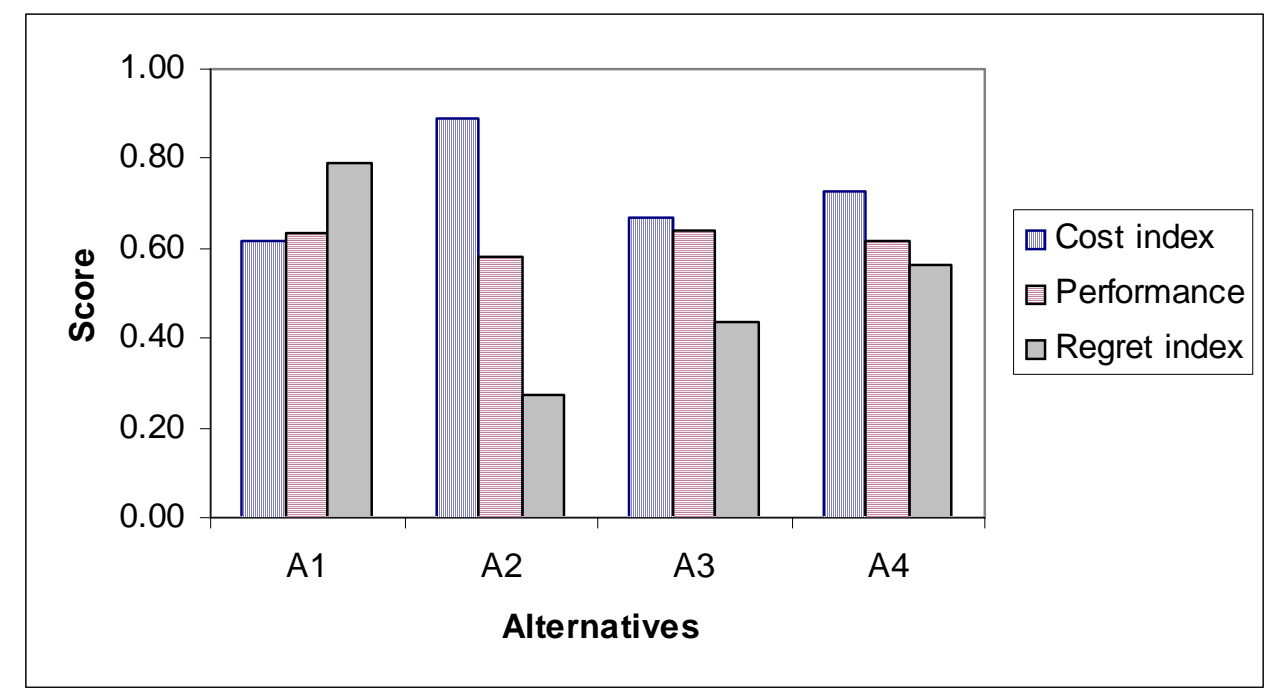

Figure 3. Comparison of the four alternatives in Table IX. High values are preferred.

\section{CONCLUDING REMARKS}

This paper is motivated by the evidence from real-life experiences and laboratory studies that:

1. Anticipated regret and rejoicing are important considerations in decision making under both uncertainty and certainty.

2. People often tend to choose "balanced choices" rather than "optimal choices", which is in conflict with classical EUT and today's typical MCDA.

3. Good DMs and analysts desire reasonably adequate information and visibility into the alternatives rather than automatic decision making based on a single number.

The RDRM and its integration within a cost-utility-regret analysis are proposed and developed as an improved MCDA method that adequately addresses the above issues from both the normative and descriptive perspectives. The applicability and usefulness of the proposed approach for complex trade-off studies is demonstrated using a hypothetical but realistic aircraft selection problem.

The RDRM is a variant of the regret theory developed by Bell and Loomes and Sugden for economic decisions under uncertainty. The level of regret (and/or rejoicing) that a person experiences is assumed to depend on the absolute values rather than simply the differences of the utilities of the chosen and forgone alternatives. It is mathematically modeled as the difference of two Wymore scoring functions. The utilities of the compared alternatives are coupled through the anticipated regret-rejoicing caused by the forgone alternatives. This is a major difference with classical utility theory. RDRM is a realistic predictive descriptor of decision making under certainty. It explains some rational behaviors that conventional MAUA, RT-B/LS, and AHP cannot. It ensures (1) transitivity of the alternatives under pairwise comparisons, and (2) rank reversal consistent with observed rational preference reversal. 
CURA is proposed as an extension of cost-utility analysis that incorporates regret as a decision element. Each alternative is characterized in terms of its monetary cost, an aggregate performance/effectiveness utility, and a regret measure. A high level of regret is an indication that the alternative is not a balanced choice. Complex situations are thereby reduced to a manageable decision problem. CURA provides the information that a good DM needs to (1) rationally trade-off some cost and/or performance to avoid high levels of regret, (2) protect against decision traps associated with human intuition [Russo and Schoemaker, 1990; Sterman, 2000: 30 and 38], and (3) avoid the fallacies and pitfalls of reducing decisions to a single number [Roy, 1999; Brown, 1999; Kujawski, 2003]. CURA can readily be extended to incorporate rejoicing for DMs who value it. The final decision it is left to the DM; it is not the automatic result of a theoretical model that produces a "best choice" based on a single number.

Researchers and practitioners of decision theory are increasingly accepting that emotions influence decisions and that in many situations individuals who act rationally do not follow the rules of classical EUT. People often tend to choose "balanced choices" rather than the "optimal choice" provided by today's typical MAUA. MCDA under certainty, from both the normative and descriptive perspectives, should be a mathematically and logically valid tool that aids DMs select alternatives that best balance cost, performance, and rationale emotions of regret and rejoicing. In this paper we propose an improved MCDA approach that integrates systems thinking, valid mathematical models, real-world experience, and behavioral psychology. We consider it a springboard to develop and implement improved decision methods and practices for individual and group trade-off studies under certainty and uncertainty where psychological influences play an important part [Scott and Antonsson, 2000]. Trade-off studies are a key function of systems engineering [Edwards, 2001]. Systems engineers can and should play an important part in the development of MCDA methods that are better predictor descriptors and hence more rational than today's approaches that are largely based on classical EUT. This is an interesting time for decision theorists and analysts. Sage's assessment of the field is as appropriate today as when he wrote [Sage, 1981: 642]

"The literature in this area is enormous. But there is the need for effort to integrate it from the perspective of systems engineering..."

\section{APPENDIX A. Proofs of Some Rank Reversal Properties of RDRM}

Theorem 1. RDRM satisfies the property of "independence of dominated alternatives".

Proof. Consider a set and two of its alternatives with preference $A_{i} \succ A_{j}$.

1. Adding an alternative $A_{k}$ that is dominated by $A_{i}$. From Eq (5) this does increase the level of regret associated with the choice of $A_{i}$ because $u_{k j} \leq u_{i j}$ for all criteria $j=1, \ldots, m$ with the strict inequality being satisfied for at least one criterion. From Eq. (5) the level of regret associated with the choice of $A_{j}$ increases if $A_{k}$ dominates $A_{i}$ for one or more criteria. The RDRM utility of $A_{i}$ therefore remains unchanged while the RDRM utility of $A_{j}$ is reduced by the increase in the 
level of regret. The preference $A_{i} \succ A_{j}$ therefore remains unchanged.

2. Dropping an alternative $A_{k}$ that is dominated by $A_{j}$. The proof that preference $A_{i} \succ A_{j}$ remains unchanged is similar to the previous one and therefore omitted.

Theorem 2. RDRM satisfies the property of "no imposed rank reversal".

Proof. From Eq. (5) it follows that rank reversal in RDRM depends on the regret-building function. The RDRM regret-building function in Eq. (6) has two parameters that allow to model levels of regret experienced by different individuals. In RDRM the occurrence of rank reversal then depends on the changes in the levels of regret experienced by an individual as a result adding or dropping an alternative.

Theorem 3. RDRM satisfies the property of "negative side effects associated with inferior substitutions".

Proof. Without loss of generality we consider alternative $A_{k}$ and replace it with $\mathrm{A}_{\mathrm{k}}{ }_{\mathrm{k}}$ where only criteria $C_{j}$ is reduced; i.e. classical utilities $u_{k j}<u^{*}{ }_{k j}$. The only possible impact on alternative $A_{i}$ is to reduce its associated level of regret. The impact is obtained using Eq. (5):

$$
\begin{aligned}
R_{i}^{*}-R_{i}=\left(\frac{1}{n-1}\right) * \mathrm{w}_{\mathrm{j}} & {\left[\max \left(\mathrm{G}\left(1-\mathrm{u}_{\mathrm{ij}}\right)-\mathrm{G}\left(1-\mathrm{u}_{\mathrm{kj}}\right), 0\right)\right.} \\
- & \left.\max \left(\mathrm{G}\left(1-\mathrm{u}_{\mathrm{ij}}\right)-\mathrm{G}\left(1-\mathrm{u}_{\mathrm{kj}}^{*}\right), 0\right)\right] .
\end{aligned}
$$

The RDRM utility is obtained by subtracting the regret level from the classical utility. There are three different cases to consider and we apply Eq. (A.1) to each.

(1) $u_{i j}>u_{k j}$. The RDRM utility of $A_{i}$ is unchanged,

$$
\mathrm{U}^{*}{ }_{\mathrm{i}}=\mathrm{U}_{\mathrm{i}} \text {. }
$$

(2) $\mathrm{u}_{\mathrm{kj}}<\mathrm{u}_{\mathrm{ij}}<\mathrm{u}_{\mathrm{kj}}$. The RDRM utility of $\mathrm{A}_{\mathrm{i}}$ is increased by eliminating the regret from $\mathrm{A}_{\mathrm{k}}$ and there is no regret from $A *_{k}$ :

$$
\mathrm{U}_{\mathrm{i}}^{*}-\mathrm{U}_{\mathrm{i}}=\left(\frac{1}{n-1}\right) * \mathrm{w}_{1} *\left[\mathrm{G}\left(1-\mathrm{u}_{\mathrm{ij}}\right)-\mathrm{G}\left(1-\mathrm{u}_{\mathrm{kj}}\right)\right] .
$$

(3) $u_{i j}<u_{k j}$ and $u_{i j}<u^{*}{ }_{k j}$. The RDRM utility of $A_{i}$ is increased by reducing the regret from $A_{k}$ to $\mathrm{A}_{\mathrm{k}}{ }_{\mathrm{k}}$ :

$$
\mathrm{U}_{\mathrm{i}}^{*}-\mathrm{U}_{\mathrm{i}}{ }^{=}\left(\frac{1}{n-1}\right){ }^{*} \mathrm{w}_{\mathrm{j}} *\left[\mathrm{G}\left(1-\mathrm{u}_{\mathrm{kj}}^{*}\right)-\mathrm{G}\left(1-\mathrm{u}_{\mathrm{kj}}\right)\right]
$$

We now consider two alternatives $A_{i} \succ A_{m}$ and the substitution of $A^{*}{ }_{k}$ for $A_{k}$ with the classical utilities related as follows: $u_{m j}<u_{i j}, u_{m j}<u_{k j}$, and $u_{m j}<u_{k j}^{*}$. The RDRM utility of $A_{i}$ is unchanged and the RDRM utility of $\mathrm{A}_{\mathrm{m}}$ is increased in accordance with Eq. (A.2c). Rank 
reversal will occur if $U_{i}^{*}<U^{*}$ which using the above results reduces to

$$
\mathrm{U}_{\mathrm{i}}-\mathrm{U}_{\mathrm{m}}<\left(\frac{1}{n-1}\right){ }^{*} \mathrm{~W}_{\mathrm{j}}{ }^{*}\left[\mathrm{G}\left(1-\mathrm{u}_{\mathrm{kj}}^{*}\right)-\mathrm{G}\left(1-\mathrm{u}_{\mathrm{kj}}\right)\right]
$$

This inequality is satisfied for select values of the classical utilities and parameters of the RDRM regret-generating function $\mathrm{G}(\cdot)$. A single case is sufficient to prove this theorem. The analyses of the other situations that may lead to rank reversal are similar and are omitted.

Note on proofs for RT-B/LS properties. The proofs that RT-L/BS satisfies Properties 1, 2, and 3 are similar to the RDRM ones and are therefore omitted.

\section{APPENDIX B. An Example of Irrational Rank Reversal in AHP}

Consider the example and analysis in Tables Xa and Xb based on Dyer's work [1990a: 252] using the original AHP. Table Xa provides the ranking for the initial set of three alternatives, A2 $\succ \mathrm{A} 1 \succ \mathrm{A} 3$. Alternative A4, which is dominated by A2, is added to the set. The alternatives are compared in Table $\mathrm{Xb}$. The ranking is now $\mathrm{A} 1 \succ \mathrm{A} 2 \succ \mathrm{A} 4 \succ \mathrm{A} 3$.

Table Xa. Initial set of three alternatives for illustrating irrational rank reversal

\begin{tabular}{|c|r|r|r|c|}
\cline { 2 - 4 } \multicolumn{1}{c|}{} & \multicolumn{1}{c|}{ Criteria, Utilities } & \multicolumn{1}{c}{} \\
\hline Alts. & \multicolumn{1}{c|}{ C1 } & \multicolumn{1}{c}{ C2 } & \multicolumn{1}{c|}{ C3 } & Rank \\
\hline A1 & 0.1 & 0.9 & 0.8 & 2 \\
A2 & 0.9 & 0.1 & 0.9 & 1 \\
A3 & 0.1 & 0.1 & 0.1 & 3 \\
\hline Weights & $1 / 3$ & $1 / 3$ & $1 / 3$ & \multicolumn{1}{c}{} \\
\cline { 1 - 3 } & & &
\end{tabular}

Table Xb. The set of four alternatives after the addition of A4

\begin{tabular}{|c|c|c|c|c|}
\hline & Criteria, & Utilities & & \\
\hline Alts. & $\mathrm{C} 1$ & $\mathrm{C} 2$ & C3 & Rank \\
\hline A1 & 0.1 & 0.9 & 0.8 & 1 \\
\hline A2 & 0.9 & 0.1 & 0.9 & 2 \\
\hline A3 & 0.1 & 0.1 & 0.1 & 4 \\
\hline A4 & 0.8 & 0.1 & 0.8 & 3 \\
\hline Weights & $1 / 3$ & $1 / 3$ & $1 / 3$ & \\
\hline
\end{tabular}

Based on intuition and experience, a rational individual who chooses A2 should not experience any additional regret because of A4. We therefore expect that the "before" and "after" utilities of A2 are identical. In contrast, a rational individual who chooses A1 may experience an increase in his anticipated regret because A4 dominates A1 on criterion C1. Most people would agree that the predicted AHP rank reversal is in conflict with the notion of rational 
behavior. Based on a literature search, this appears to be a new perspective on the shortcomings of rank reversal in AHP.

\section{ACRONYMS}

$\begin{array}{ll}\text { AHP } & \text { Analytic Hierarchy Process } \\ \text { CAIV } & \text { Cost as An Independent Variable } \\ \text { CLAU } & \text { Conventional Linearly Additive Utility } \\ \text { CUA } & \text { Cost-Utility Analysis } \\ \text { CURA } & \text { Cost-Utility-Regret Analysis } \\ \text { DM } & \text { Decision Maker } \\ \text { EUT } & \text { Expected Utility Theory } \\ \text { MAUA } & \text { Multi-Attribute Utility Analysis } \\ \text { MCDA } & \text { Multi-Criteria Design Analysis } \\ \text { MUF } & \text { Multi-attribute Utility Function } \\ \text { RDRM } & \text { Reference-Dependent Regret Model } \\ \text { RT-B/LS } & \text { Regret Theory of Bell and Loomes and Sugden }\end{array}$

\section{ACKNOWLEDMENTS}

The author wants to thank Evangelous Triantaphyllou and three anonymous referees for constructive comments that he incorporated as valuable contributions in the paper.

\section{REFERENCES}

K.J. Arrow, Social Choice and Individual Values, John Wiley and Sons, New York, 1951.

J.D. Barrow, Impossibility: The Limits of Science and the Science of Limits, Oxford University Press, Oxford, 1998.

D.E. Bell, Regret in Decision Making Under Uncertainty, Operations Research, vol. 30, 1982, pp. 961-981.

D.E. Bell, Disappointment in Decision Making Under Uncertainty, Operations Research, vol. 33, 1985, pp. 1-27.

D. Bouyssou and M. Pirlot, "Conjoint Measurement Without Additivity and Transitivity," in Advances in Decision Analysis, N. Meskens and M, Roubens (Editors), Kluwer Academic Publishers, Dordrecht, 1999, pp. 13-29.

R.V. Brown, How Misused Decision Aids Mislead Policy Makers, 1999, http://www.mccombs.utexas.edu/faculty/jim.dyer/DA_WP/WP000001.pdf.

T.R. Browning and D.A. Hillson, A Quantitative Framework for Multi-Dimensional Risk and Opportunity Management, 2004, http://sbufaculty.tcu.edu/tbrowning/publications5.htm.

D.M. Buede, The Engineering Design of Systems, John Wiley and Sons, New York, 2000. 
R.T. Clemen and T. Reilly, Making Hard Decisions with Decision Tools, Duxbury, Pacific Grove, 2001.

J.L. Corner and C.W. Kirkwood, Decision Analysis Applications in the Operations Research Literature, 1970-1989, Operations Research, vol. 39, 1991, pp. 206-219.

R.P. Cubitt, A. Munro, and C. Starmer, Testing Explanations of Preference Reversal, The Economic Journal, vol. 114, 2004, pp. 709-726.

A.R. Damasio, Descartes' Error: Emotion, Reason, and the Human Brain, Avon, New York, 1995.

J. Daniels, P. Werner, and T. Bahill, Quantitative Methods for Tradeoff Analyses, Systems Engineering, vol. 4, 2001, 190-211.

I. Das, A Preference Ordering Among Various Pareto Optimal Alternatives, Structural Optimization, vol. 18, 1999, pp. 30-35.

E. Diecidue and P.P. Wakker, On the Intuition of Rank-Dependent Utility, Journal of Risk and Uncertainty, vol. 23, 2001, pp. 281-298.

J.S. Dyer, Remarks on the Analytic Hierarchy Process, Management Science, vol. 36, 1990a, pp. 249-258.

J.S. Dyer, A Clarification of "Remarks on the Analytic Hierarchy Process, Management Science", vol. 36, 1990b, pp. 274-275.

B. Edwards (Editor), A Systems Engineering Primer for Every Engineer and Scientist, LBNL/PUB-3164, 2001.

P.T. Fishburn, Nontransitive Preferences in Decision Theory, Journal of Risk and Uncertainty, vol. 4, 1991, pp. 113-134.

G. Friedman, The intransitivity of pairwise comparisons even with a single rational decision maker or: Homomorphisms from allegedly paradoxical dice to decision-making in the military, business and sports world, Presentation to the NSF Decision-Based Design Workshop, Long Beach, January 5, 1999, http://dbd.eng.buffalo.edu/papers/friedman.html.

P.T. Harker and L.G. Vargas, Reply to "Remarks on the Analytic Hierarchy Process," Management Science, vol. 36, 1990, pp. 269-273.

R. Hastie and R.M. Dawes, Rational Choice in an Uncertain World, Sage Publications, Thousand Oaks, 2001.

G.A. Hazelrigg, Systems Engineering: An Approach to Information-based Design, Prentice Hall, Upper Saddle River, 1996.

J. L. Hennesy and D. A. Patterson, Computer Architecture: a Quantitative Approach, Morgan

Kaufmann Publishers, San Mateo, 1990.

D. Kahneman and A. Tversky, Prospect Theory: An Analysis of Decisions under Risk, Econometrica, vol. 47, 1979, pp. 313-327.

R.L Keeney and H. Raiffa, Decisions with Multiple Objectives: Preferences and Value Tradeoffs, John Wiley \& Sons, New York, 1976.

C.H. Kepner and B.B. Tregoe, The Rational Manager: A Systematic Approach to Problem Solving and Decision Making, Kepner-Tregoe, Princeton, 1965.

G. Klein, Intuition at Work, Random House, New York, 2003.

E. Kujawski, Multi-Criteria Decision Analysis: Limitations, Pitfalls, and Practical Difficulties, CD ROM Proceedings $13^{\text {th }}$ Annual International Symposium INCOSE 2003, pp. 1169-1176.

F. A. Lootsma, Fuzzy Logic for Planning and Decision Making, Kluwer Academic Publishers, Dordrecht, 1997.

F. A. Lootsma, Revisions of Basic Concepts in Decision Analysis, INFORMS Spring 2000 
Meeting, http://www.informs.org/Conf/SaltLake2000/TALKS/TC02.html

G. Loomes and R. Sugden, Regret Theory: An Alternative Theory of Rational Choice under Uncertainty, The Economic Journal, vol. 92, 1982, pp. 805-824.

G. Loomes and R. Sugden, Disappointment and Dynamic Consistency in Choice under Uncertainty, The Review of Economic Studies, Vol. 53, No. 2, 1986, pp. 271-282.

R.D. Luce and R. Raiffa, Games and Decisions: Introduction and Critical Survey, John Wiley \& Sons, New York, 1958.

R. D. Luce, Where Does Subjective Expected Utility Fail Descriptively?, Journal of Risk and Uncertainty, vol. 5,1992, pp. 5-27.

B.A. Mellers, Choice and the Relative Pleasure of Consequences, Psychological Bulletin, vol. 126, 2000, pp. 910-924.

M. Mollaghasemi and J. Pet-Edwards, Making Multiple-Objective Decisions, IEEE Computer Society Press, Los Alamitos, 1997.

J. Quiggin, A Theory of Anticipated Risk, Journal of Economic Behavior and Organization, vol. 3, 1982, pp. 323-343.

D.R. Petitti, Meta-Analysis, Decision Analysis, and Cost-Effectiveness Analysis: Methods for Quantitative Synthesis in Medicine, Oxford University Press, Oxford, 1994.

S. Plous, The Psychology of Judgment and Decision Making, McGraw-Hill, New York, 1993.

J.C. Pomerol and S. Barba-Romero, Multicriterion Decision in Management: Principles and Practices, Kluwer Academic Publishers, Boston, 2000.

B. Roy, "Decision-Aiding Today: What Should We Expect," in Multicriteria Decision Making: Advances in MCDM Models, Algorithms, Theory, and Applications, T. Gal, T.J. Stewart, and T. Hanne (Editors), Kluwer's International Series, Dordrecht, 1999, pp. 1.1-1.35.

J.E. Russo and P.J.H. Schoemaker, Decision Traps: The Ten Barriers to Brilliant DecisionMaking and How to Overcome Them, Simon \& Schuster, New York, 1990.

D.G. Saari, Bad Decisions: Experimental Error or Faulty Decision Procedures?, Invited tutorial presented at the 1999 ASME meeting, http://citeseer.ist.psu.edu/cache/papers/cs/15070/http:zSzzSzwww.math.nwu.eduzSz d_saarizSz votezSzbad-decisions.pdf/bad-decisions-experimental-error.pdf

T.L. Saaty, Fundamentals of Decision Making and Priority Theory with the Analytic Hierarchy Process, Vol. VI of the AHP Series, RWS Publications, Pittsburgh, 2000.

T.L. Saaty, An Exposition of the AHP in Reply to the Paper "Remarks on the Analytic Hierarchy Process," Management Science, vol. 36, 1990, pp. 259-268.

A. Sage, Behavioral and Organizational Considerations in the Design of Information Systems and processes for Planning and Decision Support, IEEE Transactions on Systems, Man, and Cybernetics, vol. SMC-11, 1981, pp. 640-678.

L.J. Savage, The Theory of Statistical Decision, Journal of the American Statistical Association, vol. 46, 1951, pp. 55-67.

M.J. Scott and E.K. Antonsson, Arrow's Theorem and Engineering Design Decision Making, Research in Engineering Design, vol. 1, 2000, pp. 218-228.

T.K. See and K. Lewis, Multiattribute Decision Making Using Hypothesis Testing, Proceedings of the ASME 2002 Design Engineering Technical Conferences and Computers and Information in Engineering Conference, DETC2002/DAC-34079.

P. Sen And J.B. Yang, Multiple Criteria Decision Support in Engineering Design, Springer, London, 1998.

Z. Shapira, Risk Taking: A Managerial Perspective, Russell Sage Foundation, New York, 1995. 
W.F. Sharpe, The Sharpe Ratio, The Journal of Portfolio Management, vol. 21, 1994, pp. 49-58. C. Starmer, Developments in Non-Expected Utility Theory: The Hunt for a Descriptive Theory of Choice under Risk, Journal of Economic Literature, vol. 38, 2000, pp. 332-382.

J.D. Sterman, Business Dynamics: Systems Thinking and Modeling for a Complex World, McGraw-Hill, Boston, 2000.

C. Tofallis, "Multi-Attribute Selection Using Discrete Frontier Profiling," in Advances in Decision Analysis, N. Meskens and M, Roubens (Editors), Kluwer Academic Publishers, Dordrecht, 1999, pp. 121-129.

E. Triantaphyllou, Multi-Criteria Decision Making Methods: A Comparative Study, Kluwer, Dordrecht, 2000.

A. Tversky and D. Kahneman, Advances in Prospect Theory: Cumulative Representation of Uncertainty, Journal of Risk and Uncertainty, vol. 5, 1992, pp. 297-323.

J. von Neumann and O. Morgenstern, Theory of Games and Economic Behavior, Princeton University Press, Princeton, 1953. 